\title{
A method for scheduling multi processing systems with genetic algorithm
}

\author{
Ali Pedram
}

\begin{abstract}
Methods and scheduling algorithm always a special importance in computer and solve the problem are part of their hard issues in computer science is to be considered. This is an article with the use of partition genetic algorithm classified section of the proposed a method that will be fitness the time and has convergence. This method on the basis of division and resolve and is based on a method to solve the problem multi processing scheduling systems by using this method of genetic algorithm try in the optimal time in the scheduling process in multi process systems. With this method of giving priorities to $s$ process and the atmosphere they would seek to reduce genetic algorithm that cause proposed ideal speed in finding the optimal answer.
\end{abstract}

Index Terms - Genetic Algorithms, process scheduling, multi processing systems, divide \& conquer

\section{INTRODUCTION}

Genetic algorithm programming is a technique that the genetic development as a model for solving the problem. The issue is that it must be solved algorithm entrance and reaffirms model according to a special investment code. Metric that proportion is fitness function to any candidate for the solution to evaluate most of them to be selected by random.

A genetic algorithm is a search technique in computer science to find a solution optimal and issues. One of various kinds' algorithms is genetic algorithm evolution. In the creation of the elements their biology like heredity, mutation, choosing a sudden, natural selection and the combination of inspiration. In this article by using a genetic algorithm method to solve the problem a process scheduling in a state that processor is the same speed proposal that will be subject to the proportion has done with more convergence repetitions.

In this article method offer mainland base with eight step explanation performance this method on processors with speed different performs. Fitness function accounts time is and with development time, in programmatic assimilate than method offer witness depression temporal will be actually Than elite genetic algorithm for dissolution problem scheduling several processors usage in look congest priority process and partition particular process and specialty they to Branches for divided problem to branches less than exclusivity method offer that cause becomes space search wines.

\section{PROPOSED METHOD}

After creating chromosomes, each with a similar chromosomes weight in attributed genetic algorithm, which is the same proportion is fitness function, which is based on the issue will be defined, in this issue processors maximum time completion processed are fitness function that is if less any better.

Finished time every processors with FP presentation that shortened Fitness Processor and maximum time processors with FC presentation that is shortened Fitness chromosomes. FP calculated for the total time process $\mathrm{T} 1$ to $\mathrm{T} 4$ that presentation with Fitness $=F C=M A X\left(F P_{M 1}, F P_{M 2}, F P_{M 3}\right)$ shown in Figure 1.

\section{A. Chromosomes accepted conditions}

First condition is territorial integrity: all the work is to have been sending s Processor $T=T A S K(I, P)$

Second condition is for the all Processors if $I \neq J$ then $\operatorname{TASK}(I, P) \neq \operatorname{TASK}(J, P)$ Singularity: it means there is no repetitive process.

Third condition is for the all Processors if $I \leq J$ then $\operatorname{TASK}(I, P) \leq \operatorname{TASK}(J, P)$ (Priorities)

\section{B. Cross over operation}

In this method offer, in length chromosomes a process ( gene) to sort accidental choice becomes and with process that is a group mainland base Table I On processor next exchange becomes and this exchange until end chromosomes accomplishment becomes since speed some processor not for allocated process to processor next Coefficient speed processor demands deserve in look gloomy become. Method choice chromosomes with method competitive is and two process than two processor look to look to method exchange single point hey with too exchange becomes shown in Figures 2 .

\section{Tournament selection}

In presenting proposed method of choosing a competition they use this method of choosing to briefly explain. Unlike competitive method that similar competition in the nature a small collection of chromosome to selected and competes.

Finally, in this competition based on the amount of the Fitness of one of them to victory, as a new parent in the mating pool Exchange will copy. It is essential to produce all parent $\mathrm{s}$ in new population is repeated. 


\section{Mutation operations on the processor}

Mutation operations on processor are done in this way: a process chosen a process randomly on a process queue. This process is transferred to another processor queue that shown in Figure 2-c.

\section{DESCRIPTION PROPOSED METHOD TO STEP BY STEP}

\section{A. First step of proposed method}

At first proposed method in the process with regard to the situation and the dependence that are $\mathrm{DAG}^{1}$ show that the Graph on this DAG dependence on the number and level and also tied the knot father and child are shown. DAG to parent process is not processing child process will be.

\section{B. Second step of proposed method}

This method based on the priorities with regard to the level of node and the number of you tied with reference to the high-level will be working. With regard to form Table I is T0, T1, T2, T11 nods in the level number zero and the rest are tied with the number levels with regard to form will be clear. DAG maximum depth is 3 that this parameter in proposed Formula (1).

$$
\begin{aligned}
& \text { prt }_{i}=\text { priority .of } T_{i} \\
& \text { max level }=\max \text { imum.level.of .DAG } \\
& L_{i}=\text { level.number } T_{i} \\
& T_{i}=\text { process with.number. } i \\
& \text { prtchild }{ }_{i}=\text { Total .of .Childs } T_{i} \\
& \text { refhigh } h_{i}=\text { number.of .refrence.at.high.levels } \\
& \text { prt }_{i}=\max \text { level }-L_{i}+\text { refhigh } h_{i}+\text { prtchild }
\end{aligned}
$$

\section{Third step of proposed method}

Now each node with a priority in the Table I and a descending sorted. In Formula (2) see the process, the number of processes and the number of processors and calculate number of partitions.

$$
S_{\text {number }}=\frac{n}{m}
$$

TABLE I THE PROCESS WITH THE AMOUNT OF PRIORITIES DESCENDING HAS SORTED.

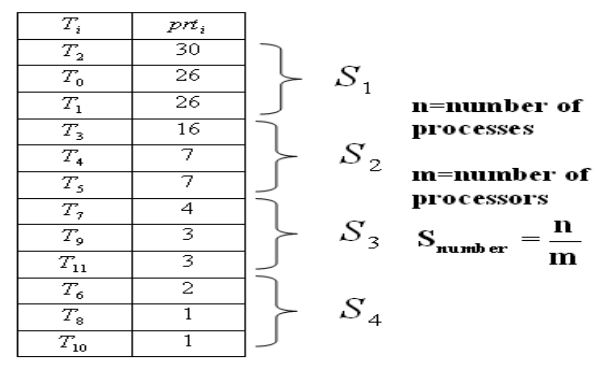

\section{Fourth step of the proposed method-partitioning}

Table I show could be partition genetic algorithm to use a classified section of the process and separates partition with the priority so that every one of the priorities of the process would be processed. The number of partitions with regard to the number of processors and process s determine. For example, if three separate processors with different rate, the number of this form to calculate sections. As a suggestion that we are the table divided into four separate sections, and genetic algorithm run to them separately hat shown in Table I and Figures 3.

Firstly has not repeated the process (chromosome gene have not repeated).

Secondly: a parent process necessarily must have similar chromosomes in before child's process. Before the start child processed It processed soon will finish the priorities of course, if the process to be sorted by descending this possibility for.

Thirdly: process all the people must be processing.

\section{E. Fifth step generate population}

Now to be allocated processes to the processors. Allocation process with regard to the priority in the table that amount was specified priorities in the queue given and processors are allocated. Strategy that in allocated process to processors use is that in the partition larger processes to faster processors and smaller partitions dedicate to process with least rate. Allocated process too in partitions to order priority branches accomplishment becomes and another strategy is allocated to random dedicate to processors.

if assume a system include three processors with speed different for example M2 with speed double M1 and M3 quadruple M1 ، after allocated a process To processor that processing time process mainland coefficient speed processor division. For example has mentioned that if a process is to be allocated M3 processors time that the process is divided into four and if a process is to be allocated M3 processing time divided into two. In a way to get FC that how much less any better.

For allocation process to processors in descending order, and the processes of the accident processors are allocated.

\section{F. Sixth step cross over and evaluation}

For cross over operation two process than processors peer to peer select and exchange accomplishment becomes that process On performance queue processors peer to peer exchange conditional that last saying became, namely process parent performance deserve All had become until process child that performance become. Deserve care had that processes existent in partitions that in lower priority perfumed until ends agreement request processes on two chromosome until end chromosome with process too partition that exchange.

similar chromosomes assessment in this way we do that produced a similar chromosomes has always been his process is based on a descending spiral her priorities sorting to credit similar chromosomes have similar chromosomes otherwise we will not be valid.

\section{G. Seventh step mutation}

In the step that parts of the previous explained implemented. Mutation in this issue to be under our proposal The exchange only one process on two execute queue different processors that this process to choose a random, and on another processor. Of course it can be an intelligent strategy for doing this linear allotted for example of a processor Porous choice and to a processor at least give carter. 


\section{H. Eight step select chromosomes}

After generation elementary population that use genetic operator such as cross over and mutation. After end any operation repeat validation chromosome repeat accomplishment becomes until chromosome by validation chromosome FC sorted that less than chromosome Next validation above. Chromosome to order validation by descending neat and also integrity and unique demands deserve observance become. Select in method offer choice competitive.

\section{SIMULATED RESULTS PROPOSED METHOD}

Programs like instrument that with the program is implemented with 99 processes and we have implemented processor with high speed coefficient of $4 \mathrm{x}$, processor with middle speed coefficient of $2 x$, processor with low speed coefficient $x$ we have the result.

In Figure 4-a we see proportion subject, after almost 2500 repeated (generate chromosome) amount to 27.1497 it seems that one another point is of course it's fitness function to a proportion approximate amount to a point to stop time will be defined in this program for all time visa process are gathered together, and the number of processor are divided into prophecy.

Competition in this red line high speed processor, Green Line shows middle speed processor and blue line slows processor with her speed and also the black line time is maximize. Three charts is the same FC.

With care to result that to hand came system several processor with method multilevel queue case test put that with processes that in sectional similar Build usage became result earned total time processing processes noses 100 until 300 one temporal with care to attitudes diverse that in queue processing agreement congests for 99 process that in three queue processing stood to hand came conclusion total time processing 99 trend with method queue multilevel for systems several processor considerate Table II with a comparison noses method offer and method multilevel queue wine strength concluded that genetic algorithm that shown in Figures 4.

TABLE II PROCESSING TIME OF THE WHOLE PROCESS S METHOD LINE WITH SOME SUPERFICIAL FOUR DIFFERENT TESTS WITH THE NUMBER OF 99 PROCESSES

\begin{tabular}{|c|c|}
\hline Fitness Function & Test number \\
\hline 29.28582 & first test \\
\hline 17.52591 & second test \\
\hline 17.17397 & third test \\
\hline 21.58629 & fourth test \\
\hline
\end{tabular}

\section{CONCLUTION}

That information has come up with this method proposal shows that the issue as a hard time complexity of with a suitable complexity solution. Because we want show to the operations by genetic algorithm better time for us. Genetic algorithm to solve the problems with the appropriate methods does not recover.

In this article proposed to solve the problem multi processing scheduling with processors that have different speed solve that with regard to the results simulator program we observed by using the divide and conquer the visa process and if increasing the number of repetitions then subject to proposed proportion will decrease.

A proposal that in case this method introduces this is that cans this use method for job scheduling that time them non linear. For example for traffic control network and user scheduling in distributed systems use than this method that has a good complexity. Of course create direct acyclic graph creation become in this method than direction measurement improvement donates for example by $A^{*}$ combine genetic algorithm method for measurement direct acyclic graph with this method basis genetic algorithm usage appearance.

\section{REFERENCES}

[1] S. William, Operating Systems: Internals and Design Principles, Prentice Hall, 2008.

[2] E. L.Lawler et al., The Traveling Salesman Problem, John Wiley \& Sons, New York, 1985.

[3] Kylie Bryant, "Genetic Algorithm and the traveling salesman problem", Hervey Mudd college, 2000.

[4] Hupt, Practical Genetic Algorithms, John Wiley, 2004.

[5] Annie S. Wu, Han Yu, Shiyuan Jin, Kuo-Chi Lin, and Guy Schiavone, "An Incremental Genetic Algorithm Approach to Multiprocessor Scheduling", Menbers IEEE, 2004.

[6] David Crafti, "A Job Shop Scheduler using a Genetic Tree Algorithm", School of Computer Science and Software Engineering Monash University Clayton, Victoria Australia, 2004. Prashanth C SaiRanga, Sanjeev Baskiyar, PhD, "A Low Complexity Algorithm for Dynamic Scheduling of Independent Tasks onto Heterogeneous Computing Systems", Department of CSSE Department of CSSEAuburn University Auburn University AL 36849 334-359-8853, ACM 2005.

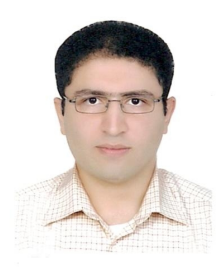

First A. Ali Pedram : Ali Pedram received his B.S in computer engineering from Islamic Azad University Tehran Branch, in 2001, the M.S. in computer engineering from IAU University Ahwaz Branch, in 2008.He is teaching Department of Branch. Computer at the Islamic Azad University Abadan

$\mathrm{He}$ is the author/co-author of 4 publications in technical journals and conferences. His research interests are in the areas of genetic algorithm, scheduling algorithms and evolutionary computing. 


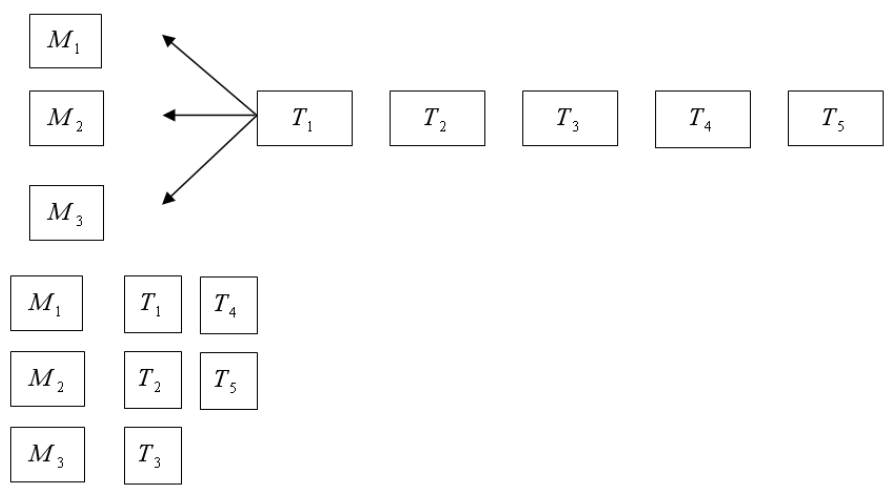

Figure 1 The allotment of process to processors and fitness calculate proportion.

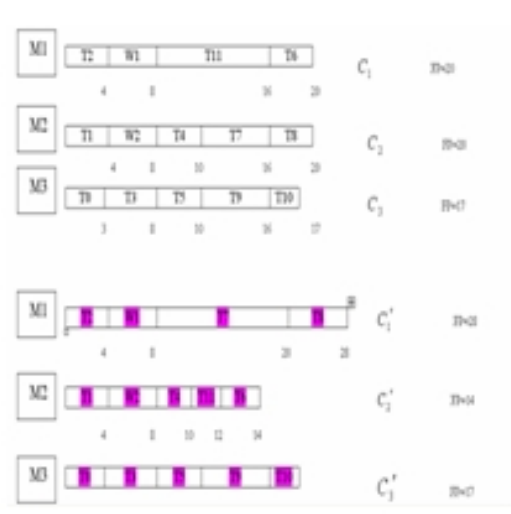

(a)
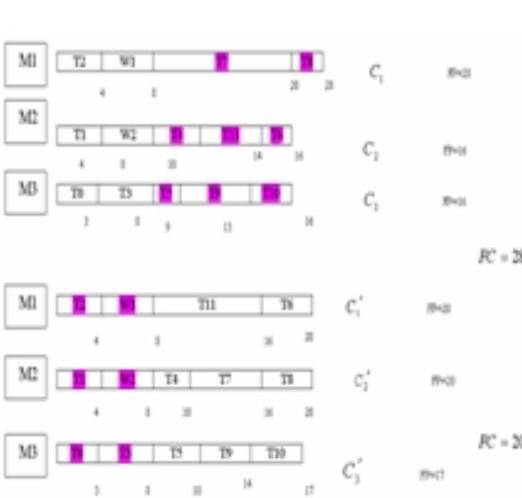

(b)
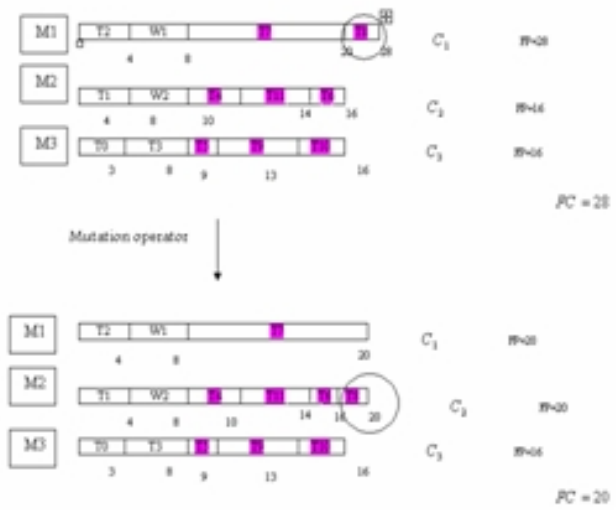

(c)

Figure 2 Chromosomes crossover before (a) and after of a single point cross over (b) then next figure show a mutation operator (c).

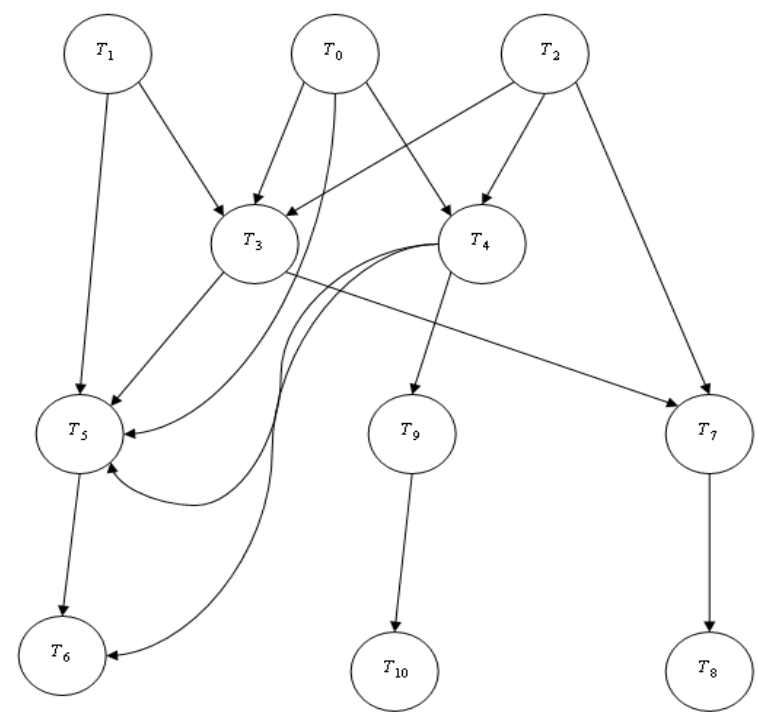

(a)
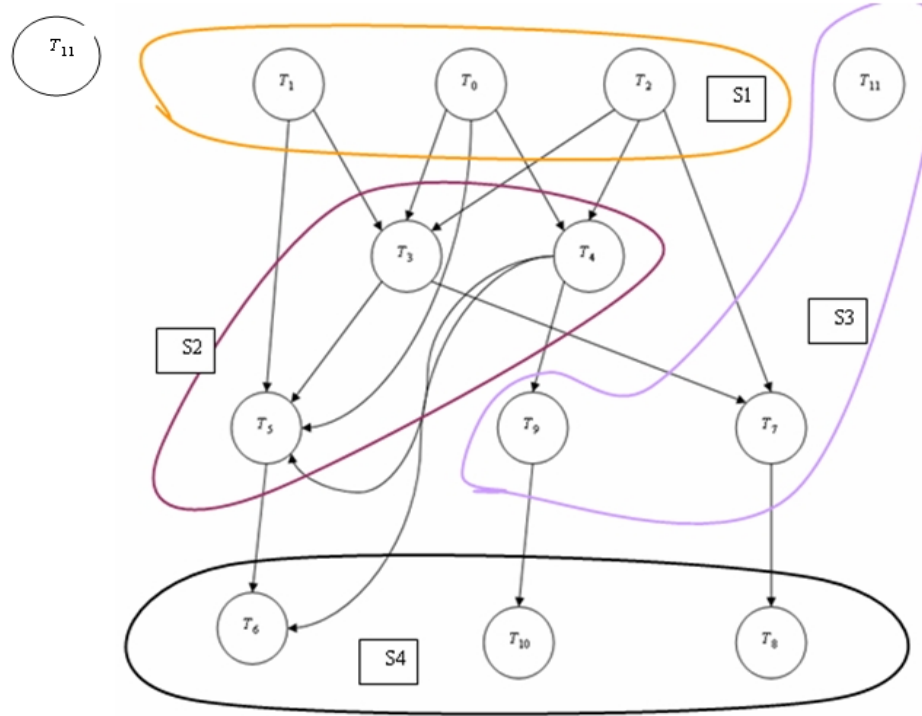

(b)

Figure 3 Create A DAG of the process (a) figure (b) show partitioning on the DAG with priority. 


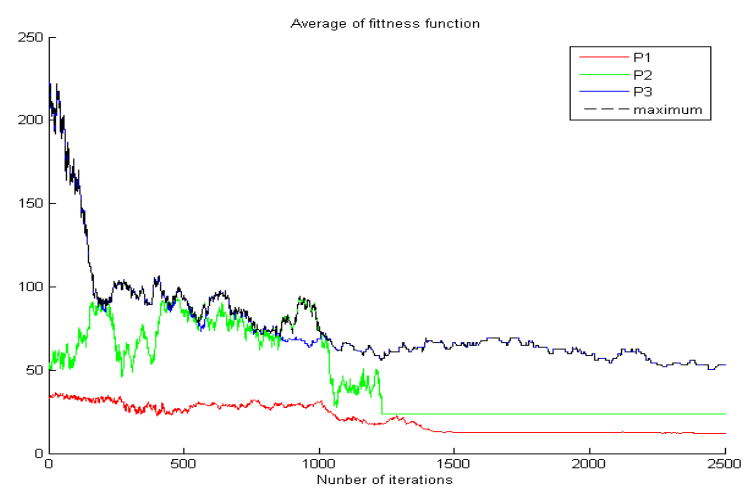

(a)

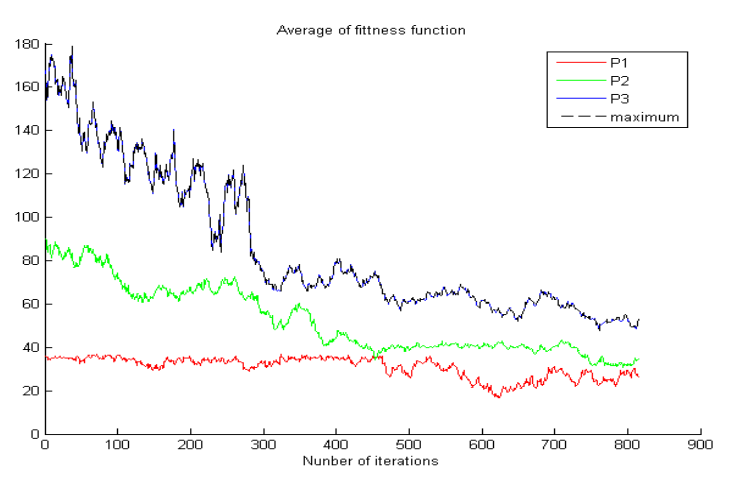

(c)

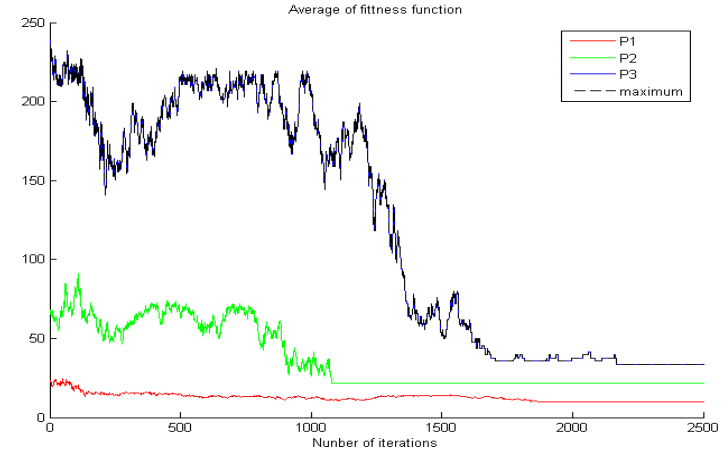

(b)

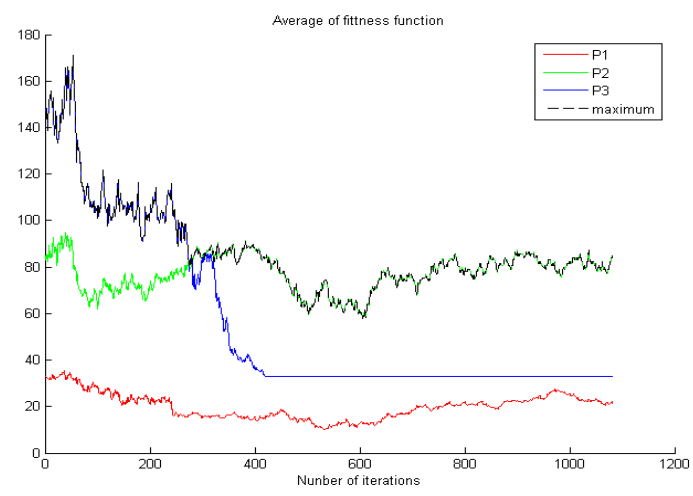

(d)

Figure 4 Processing 99 processes with 33 partitions the four tests. 